\title{
1 Significance of CSF NfL and tau in ALS
}

2 Stefanie Schreiber ${ }^{1,2}, \mathrm{MD}$; Nicola Spotorno ${ }^{2}$, PhD; Frank Schreiber ${ }^{1,2,3}$; Julio Acosta-

3 Cabronero ${ }^{2,4}$, PhD; Jörn Kaufmann"1, PhD; Judith Machts ${ }^{1,2}$, PhD; Grazyna Debska-

4 Vielhaber ${ }^{1}$, PhD; Cornelia Garz ${ }^{1,2}$; Daniel Bittner ${ }^{1,2}$, MD; Nathalie Hensiek'; Reinhard

5 Dengler ${ }^{5}$, MD; Susanne Petri ${ }^{5}$, MD; Peter J Nestor ${ }^{2,6}$, MD; Stefan Vielhaber ${ }^{1,2}$, MD

61 Department of Neurology, Otto-von-Guericke University, Leipziger Straße 44, 39120

$7 \quad$ Magdeburg, Germany

82 German Center for Neurodegenerative Diseases (DZNE) within the Helmholtz 9 Association, Leipziger Straße 44, 39120 Magdeburg, Germany

103 Institute of Control Engineering, Technische Universität Braunschweig, Hans11 Sommer-Straße 66, 38106 Braunschweig, Germany

124 Wellcome Centre for Human Neuroimaging, UCL Institute of Neurology, University 13 College London, London, UK

145 Department of Neurology, Hannover Medical School, Carl-Neuberg-Straße 1, 30625 Hannover, Germany

166 Queensland Brain Institute, University of Queensland, Brisbane 4072, Australia

17 Word count

18 Abstract

19 Manuscript (not including the abstract)

20 Title

\section{Corresponding author}

22 Stefanie Schreiber

23 Department of Neurology

24 Otto-von-Guericke University

$25 \quad$ Leipziger Straße 44
354 words

3050 words

31 characters 
39120 Magdeburg

27 stefanie.schreiber@med.ovgu.de

28 Telephone + 493916713431

29 Fax

$+493916715233$

30 Nicola Spotorno nicola.spotorno@dzne.de

$31 \quad$ Frank Schreiber frank.schreiber@med.uni-magdeburg.de

32 Julio Acosta-Cabronero jac@cantab.net

33 Jörn Kaufmann joern.kaufmann@med.ovgu.de

34 Judith Machts judith.machts@dzne.de

35 Grazyna Debska-Vielhaber grazyna.debska-vielhaber@med.ovgu.de

36 Cornelia Garz cornelia.garz@med.ovgu.de

37 Daniel Bittner daniel.bittner@med.ovgu.de

$38 \quad$ Nathalie Hensiek nathalie.hensiek@st.ovgu.de

39 Reinhard Dengler dengler.reinhard@mh-hannover.de

40 Susanne Petri petri.susanne@mh-hannover.de

$41 \quad$ Peter J Nestor p.nestor@uq.edu.au

42 Stefan Vielhaber stefan.vielhaber@med.ovgu.de

43 Statistical Analysis conducted by Stefanie Schreiber, MD, Nicola Spotorno, PhD, and Frank 44 Schreiber, Otto-von-Guericke University and DZNE Magdeburg.

45 Keywords: Amyotrophic lateral sclerosis, MRI, Cerebrospinal fluid, Diffusion tensor 46 imaging, Neurofilament light chain, Ttau, tau phosphorylated at threonine 181 (ptau), 47 ptau/ttau ratio 


\section{Author Contributions}

SS: data analysis, data interpretation, study concept and design, drafting the manuscript

51 NS, FS: data analysis, data interpretation, study concept and design, critical revision of the

52 manuscript for important intellectual content

53 JAC, JK, JM, GDV, CG, DB, NH, RD, SP: acquisition of data, critical revision of the manuscript for important intellectual content

PJN: critical revision of the manuscript for important intellectual content

56

SV: acquisition of data, data interpretation, study concept and design, drafting the manuscript, critical revision of the manuscript for important intellectual content, study supervision

SS had full access to all of the data of the study, and takes responsibility for the integrity of the data and the accuracy of the data analysis.

\section{Acknowledgements}

We thank Christa Sobetzko, Department of Neurology, Otto-von-Guericke University, Magdeburg, Germany, for data collection.

\section{Author Disclosures}

Stefanie Schreiber - Reports no disclosures

Nicola Spotorno - Reports no disclosures

Frank Schreiber - Reports no disclosures

Julio Acosta-Cabronero - Reports no disclosures

Jörn Kaufmann - Reports no disclosures

Judith Machts - Reports no disclosures

Grazyna Debska-Vielhaber - Reports no disclosures

Cornelia Garz - Reports no disclosures 
73 Daniel Bittner - Reports no disclosures

74 Nathalie Hensiek - Reports no disclosures

75 Reinhard Dengler - Reports no disclosures

76 Susanne Petri - Reports no disclosures

77 Peter J Nestor - Reports no disclosures

78 Stefan Vielhaber - Reports no disclosures

79 On behalf of all authors, the corresponding author states that there is no conflict of interest.

80 This work was supported by a grant to SS from the Otto-von-Guericke University Magdeburg

81 (grant for young scientists 2016) and by a grant to SS and SV from the Foundation of 82 Medical Research, Frankfurt / Main, Germany. 
83

84

85

86

87

88

89

90

91

92

93

94

95

96

97

98

99

100

101

102

103

104

105

106

107

\section{Abstract}

Cerebrospinal fluid (CSF) neurofilament light chain (NfL) has emerged as putative diagnostic biomarker in amyotrophic lateral sclerosis (ALS), but it remains a matter of debate, whether CSF total tau (ttau), tau phosphorylated at threonine 181 (ptau) and the ptau/ttau ratio could serve as diagnostic biomarker in ALS as well. Moreover, the relationship between CSF NfL and tau measures to further axonal and (neuro)degeneration markers still needs to be elucidated.

Our analysis included 89 ALS patients (median (range) age 63 (33-83) years, 61\% male, disease duration $10(0.2-190)$ months) and 33 age- and sex-matched disease controls (60 (32-76), 49\%). NfL was higher and the ptau/ttau ratio was lower in ALS compared to controls $(8,343(1,795-35,945) \mathrm{pg} / \mathrm{ml}$ vs. $1,193(612-2,616), \mathrm{H}(1)=70.8, \mathrm{p}<0.001$; mean [SD] 0.17 [0.04] vs. 0.2 [0.03], $F(1)=14.3, p<0.001$ ), as well as in upper motor neuron dominant (UMND, $n=10)$ compared to classic $(n=46)$ or lower motor neuron dominant ALS $(n=31$; for NfL: $16,076(7,447-35,945)$ vs. $8,205(2,651-35,138)$ vs. $8,057(1,795-34,951), Z \geq 2.5$, $p \leq 0.01$; for the ptau/ttau ratio: $(0.13$ [0.04] vs. 0.17 [0.04] vs. 0.18 [0.03], p $\leq 0.02)$. In ALS, $\mathrm{NfL}$ and the ptau/ttau ratio were related to corticospinal tract (CST) fractional anisotropy (FA) and radial diffusivity (ROI-based approach and whole-brain voxelwise analysis). Factor analysis of mixed data (FAMD) revealed a co-variance pattern between NfL (factor load 0.6), the ptau/ttau ratio (0.7), CST FA (0.8) and UMND ALS phenotype (-2.8). NfL did not relate to any further neuroaxonal injury marker (brain volumes, precentral gyrus thickness, peripheral motor amplitudes, sonographic cross-sectional nerve area), but a lower ptau/ttau ratio was associated with whole-brain gray matter atrophy and widespread white matter integrity loss. Higher NfL baseline levels were associated with greater UMN disease burden, more rapid disease progression, a 2fold to 3fold greater hazard of death and shorter survival times. 
108 The findings that higher CSF NfL levels and a reduced ptau/ttau ratio are more associated 109 with clinical UMN involvement and with reduced CST FA offer strong converging evidence 110 that both are markers of central motor degeneration. Furthermore, NfL is a marker of poor 111 prognosis, while a low ptau/ttau ratio indicates extramotor pathology in ALS. 


\section{Introduction}

113 Cerebrospinal fluid (CSF) neurofilament light chain (NfL) has emerged as putative diagnostic

114 biomarker in several neurodegenerative conditions [1, 2], such as amyotrophic lateral 115 sclerosis (ALS), and ALS patients reveal significantly higher levels compared to controls or 116 disease mimics [3-9]. CSF neurofilaments seem also to aid as a prognostic biomarker [4, 5,

$1178-11]$ and have been found to be higher in ALS patients with dominant upper motor neuron 118 (UMN) involvement $[4,5,12]$. In the meanwhile, several studies have also taken account of serum NfL which has additionally proven great potential to discriminate between ALS and controls or disease mimics [7, 13, 14]. Compared to CSF, serum neurofilament might, however, be less sensitive towards clinical and electrophysiological measures of motor neuron degeneration $[9,15]$, emphasizing the pivotal role of CSF neurofilaments when taking account of the extent of neuroaxonal damage despite less convenient sampling. With the exception of few studies focusing on the corticospinal tract's (CST) integrity applying diffusion tensor imaging (DTI) there is, however, nearly no data available how CSF neurofilaments relate to other biomarkers of peripheral (PNS) and central nervous system (CNS) neuroaxonal injury in ALS $[6,16]$. Understanding their certain biomarker associations would indeed even improve the leading candidate role of CSF neurofilaments to aid as a promising outcome measure in future ALS therapeutic trials [17].

In contrast to CSF NfL, it is a matter of debate whether CSF total tau (ttau) and tau phosphorylated at threonine 181 (ptau) could serve as diagnostic biomarker in ALS as well, and there are just as many studies reporting elevated (abnormal) or normal levels in ALS when compared to controls [12, 18-25]. Two studies further proposed a reduction of the 134 ptau/ttau ratio in ALS $[23,24]$. While CSF tau seems not to universally correlate with disease progression in ALS [12, 19, 21, 26], few studies indeed found a relationship between higher CSF ttau or ptau at baseline and worse motor function $[6,23]$. And, as for CSF NfL, with the exception of two DTI studies reporting an association between CST integrity and the 
138 ptau/ttau ratio, but not with ttau or ptau, there are no data available how tau measures relate

139 to further neuroaxonal injury markers in ALS $[6,23]$. Continuing studies are thus indeed 140 needed to determine the biomarker role of ttau and ptau in ALS.

141 We thus here conducted several analyses taking especially into account how CSF NfL, ttau, 142 ptau and the ptau/ttau ratio distribute within certain ALS subgroups, relate to further axonal 143 and (neuro)degeneration markers such as motor amplitudes or precentral gyrus thickness 144 and the ALS patients' long-term outcome using a retrospective approach. 
Methods

\section{ALS sample}

147 Our study comprised 89 ALS patients recruited from the Departments of Neurology, Ottovon-Guericke University, Magdeburg and Hannover Medical School, Hannover, Germany.

149 Patients were diagnosed by one of two experienced neurologists (S.V., S.P.) according to

150 the revised El Escorial criteria comprising the assessment of the number of regions (bulbar, 151 thoracic, upper limb, lower limb) with UMN (clinically) or lower motor neuron (LMN) 152 involvement (clinically or via electromyography) [27]. Similar to previous studies, we also 153 included patients presenting with LMN signs only ("suspected ALS") [14]. The Penn UMN score was recorded to assess the UMN disease burden in the bulbar segment as well as in

155 each of the four limbs [28] (see Supplemental). ALS clinical phenotypes were classified in 156 line with operational definitions as specified previously [29, 30] (see Supplemental). At 157 baseline patients underwent a clinical and diagnostic work-up (ALS functional rating scale (ALSFRS-R) total score, genetic testing, CSF measures of NfL, ttau, ptau, total protein and the CSF albumin/serum albumin ratio $\left(Q_{a l b} \times 10^{-3}\right)$; for methodological details regarding CSF measurements and the performance of the NfL assay see Supplemental and Supplemental Table 1). Measures of neuroaxonal injury comprised those PNS and CNS markers

162 commonly found to be altered in ALS: median and ulnar nerve compound motor action 163 potential (CMAP) amplitudes [31, 32] and sonographic cross-sectional nerve area (CSA) [29, 164 33] (for methodological details of PNS measures see [29]), precentral gyrus thickness, cortical and subcortical cerebral gray matter (GM) volumes and CST DTI metrics (e.g. fractional anisotropy (FA)) [34-37]. Disease duration was the time in months between symptom onset and a patient's baseline visit. Disease progression rate (DPR) was

168 determined as (48-ALSFRS-R)/disease duration (points per month). Patients underwent 169 follow-up ALSFRS-R measurements within a mean [SD] time interval of 6 [8] months. Please 170 see the Supplemental and Supplemental Figure 1 for the detailed demonstration of the 171 availability of all measures. 
173 Cross-sectional CSF NfL, ttau and ptau measures were additionally conducted in a hospitalbased cohort of 33 neurologic patients (non-motor neuron disease controls), comprising cases with non-specific complaints who underwent lumbar puncture in terms of a diagnostic work up to rule out any neurologic condition. None of those disease controls suffered from any neuromuscular disorders (i.e. peripheral polyneuropathies, muscle or motor neuron disease) nor did they display any specific abnormalities on the neurological exam. CSF NfL data were available in all subjects, while tau measures have been conducted in 16 out of the 33 control cases only (please see Supplemental Table 2 for further details).

\section{Standard protocol approvals, registrations, and patient consents}

The study was approved by the local ethics committee (No. $150 / 09$, No. $07 / 17$, No. 16 / 17), and all subjects gave written informed consent.

\section{T MRI measures of the brain}

All MRI sessions were performed on the same Siemens Verio 3 T system (Siemens Medical Systems, Erlangen, Germany) at the same site (Magdeburg), and all patients underwent exactly the same MRI protocol. 3D MPRAGE images were acquired (for bilateral precentral gyrus thickness, GM (GMV) and white matter (WMV) and total brain (TBV) volumes). Diffusion MRI data were used to compute the maps of DTI scalars (FA, mean diffusivity $(\mathrm{MD})$, radial diffusivity (RD), axial diffusivity (AD)). Applying tract-based spatial statistics [38] whole-brain regression analysis with white matter hyperintensities (WMH) as covariate of no interest (assessed in a T2-weighted FLASH sequence according to the Fazekas scale [39]) was conducted (with the Randomise tool version 2.9 available in FSL, 5000 permutations, threshold-free cluster enhancement (TFCE), 2D optimization for tract-based DTI analysis). Individual median values of bilateral CST DTI scalars were additionally extracted (region of interest (ROI) analysis). For the in-depth demonstration of all imaging analyses see the

\section{Supplemental.}




\section{Statistics}

199 Gaussian distribution of data was assessed using the Shapiro-Wilk test. For group 200 comparisons, for non-normally distributed data Kruskal-Wallis one-way analysis of variance 201 (ANOVA) with post hoc pairwise Mann-Whitney $U$ testing, and for normally distributed data 202 ANOVA with Bonferroni post hoc testing was conducted. Relationship between distinct 203 variables was calculated using bivariate correlations. Left- and right-sided CMAP amplitude 204 and CSA measures were averaged, as there were no side differences. Compared to the left 205 side, the right motor cortex was significantly thinner $(Z=-5.0, p<0.001$, Wilcoxon signed-rank 206 test), which is a common finding in ALS [40]; left- and right-sided measures were thus 207 considered separately.

208 We then applied a factor analysis for mixed (quantitative and qualitative) data (FAMD) using 209 FactoMineR version 1.27 [41] to capture co-variance patterns between distinct measures 210 related to CSF NfL. We included CSF NfL, the ptau/ttau ratio, CST FA (which is the most 211 sensitive DTI metrics in ALS [42]) and ALS phenotype into that model and extracted 1 component with an eigenvalue $>1$, which explained $40 \%$ of the variance in the data.

213 Random intercept mixed effects linear models with CSF NfL (ttau, ptau, ptau/ttau ratio) 214 median-split (main effect) and time (disease duration) in months (main effect) were 215 calculated to assess CSF NfL (ttau, ptau, ptau/ttau ratio) $\times$ time interaction effects on 216 longitudinal ALSFRS-R total score, and estimates (e) are given. In addition, Kruskal-Wallis 217 one-way ANOVA or ANOVA was calculated to assess group effects of slow (averaged 218 ALSFRS-R points lost per month $<0.4$ from disease onset to last available ALSFRS-R), 219 intermediate $(\geq 0.4, \leq 1.4)$ and fast $(>1.4)$ disease progressors [43] on baseline CSF NfL (ttau, 220 ptau, ptau/ttau ratio).

221 Cox proportional hazard models giving the hazard ratio $(\operatorname{Exp}(B))$ and Kaplan-Meier analysis 222 using a pairwise log rank test were conducted to compare survival rates and times between 
223 ALS patients revealing low, medium or high CSF NfL (ttau, ptau, ptau/ttau ratio) levels 224 (terciles) at baseline, and censoring was done at the date of the last follow-up.

225 P-values $<0.05$ were deemed to be statistically significant. Analyses were performed using 226 the IBM SPSS Statistics 23.0 software. 


\section{Results}

228

Sample

229

Table 1 demonstrates the demographics and the clinical data of the whole sample.

230 Supplemental Table 3 depicts the demographics and clinical data separately for the ALS 231 phenotypes.

232

233

234

235

236

237

238

239

240

241

242

243

244

245

246

247

248

249 250

\section{CSF NfL, ttau, ptau and ptau/ttau ratio in controls and ALS}

CSF NfL lacked symmetry (controls: $\mathrm{D}(33)=0.9, \mathrm{p}=0.02$; $\mathrm{ALS}: \mathrm{D}(89)=0.9, \mathrm{p}<0.001$ ) but revealing a significant positive skew distribution instead with most measures clustering at the lower end of the scale (controls: $z_{\text {skewness }}=2.6, p<0.01$; ALS: $z_{\text {skewness }}=5.6, p<0.001$; Figure 1A). In ALS, distribution was the same for CSF ttau and ptau (ttau: $D(88)=0.9, p<0.001$, $z_{\text {skewness }}=4.4, p<0.001 ;$ ptau: $D(88)=0.9, p<0.001, z_{\text {skewness }}=4.1, p<0.001$ ), while the ptau/ttau ratio was normally distributed $\left(D(88)=1.0, p=0.4, z_{\text {skewness }}=1.5, p>0.05\right)$. In controls all CSF tau measures were normally distributed (ttau: $\mathrm{D}(14)=0.9, \mathrm{p}=0.5, \mathrm{z}_{\text {skewness }}=-0.7, \mathrm{p}>0.05$; ptau: $D(14)=1.0, p=1.0, z_{\text {skewness }}=-0.04, p>0.05$; ptau/ttau ratio: $D(14)=0.9, p=0.3, z_{\text {skewness }}=1.3$, $p>0.05$ ) (Figure 1B).

In ALS compared to controls, NfL was higher (median (range) $8,343(1,795-35,945) \mathrm{pg} / \mathrm{ml}$ vs. $1,193(612-2,616), H(1)=70.8, p<0.001)$ and the ptau/ttau ratio was lower (mean [SD] $0.17[0.04]$ vs. $0.2[0.03], F(1)=14.3, p<0.001$; Figure 1A\&B). There were no group differences for ttau and ptau (ALS vs. controls, ttau: 236 (96-666) pg/ml vs. 260 (122-373), ptau: 40 (17-99) pg/ml vs. $53(20-80))$.

In ALS, NfL was related to $Q_{a l b}$ ( $r h o=0.2, p=0.04$ ), and ttau and ptau were related to age (rho $=0.4, p<0.001$, respectively). There was no association with sex, disease duration, or onset site; NfL did relate to the ptau/ttau ratio ( $r h o=-0.4, p<0.001$; Figure $1 \mathrm{C}$ ), but not to ttau and ptau. 


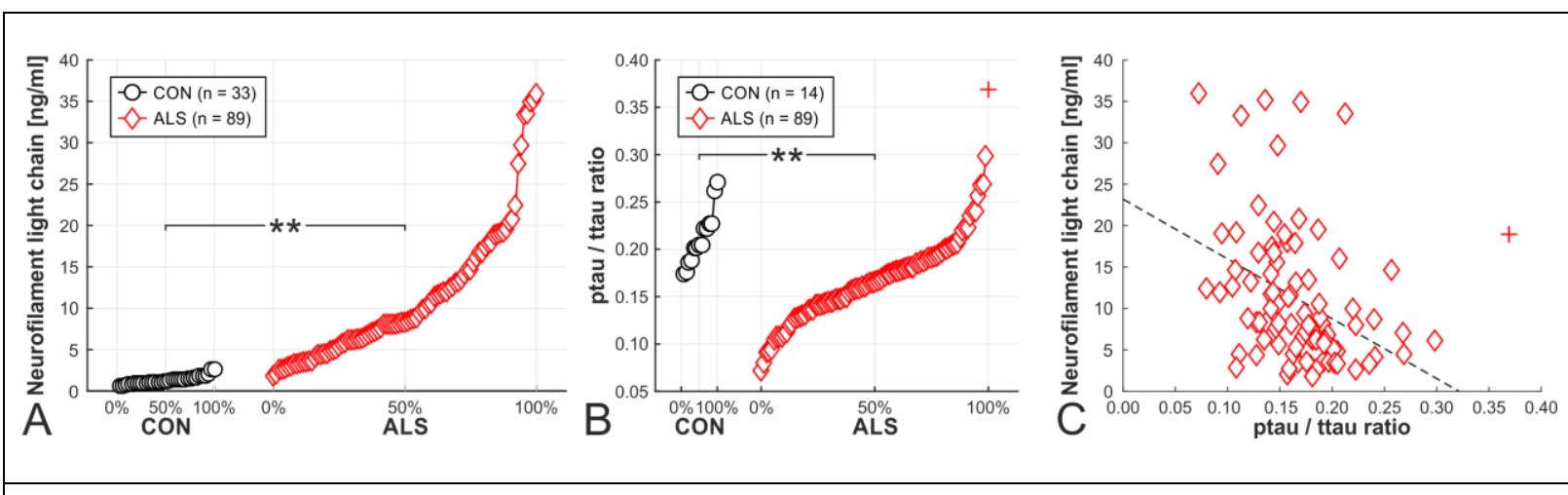

Figure 1. Quantile function of CSF NfL values and the ptau/ttau ratio within the samples under investigation

Graph demonstrates CSF NfL concentrations (A) and the ptau/ttau ratio (B) in controls $(\mathrm{CON})$ and ALS. ALS patients compared to controls revealed significantly higher CSF NfL concentrations and a significantly lower ptau/ttau ratio. C demonstrates the significant relationship between CSF NfL and the ptau/ttau ratio in ALS. ${ }^{* *} \mathrm{p} \leq 0.001$.

251 There was a significant effect of clinical phenotype on CSF NfL $(H(2)=7.9, p=0.02)$ and on 252 the CSF ptau/ttau ratio $(F(2)=6.6, p=0.002)$. Pairwise comparisons revealed group 253 differences between classic and upper motor neuron dominant (UMND) (NfL: Z=2.5, $p=0.01$; 254 ptau/ttau ratio: $\mathrm{p}=0.02$ ) or lower motor neuron dominant (LMND) and UMND ALS (NfL: $255 \mathrm{Z}=2.6, p=0.008$; ptau/ttau ratio: $\mathrm{p}=0.001$ ), with UMND compared to classic or LMND patients 256 displaying higher NfL $(16,076(7,447-35,945)$ vs. 8,205 (2,651-35,138) vs. 8,057 (1,795257 34,951); Figure 2A) and a lower ptau/ttau ratio (0.13 [0.04] vs. 0.17 [0.04] vs. 0.18 [0.03]; 258 Figure 2C). In PLS, NfL was lower than in ALS $(7,043(6,454-7,632))$, and the ptau/ttau ratio 259 was rather similar to the ratio in classic/LMND, but higher than in UMND ALS (0.16 [0.02]); 260 as there were only 2 PLS cases (Table 1), they were, however, not considered for group and 261 pairwise subgroup comparisons.

262 There was, moreover, a significant relationship between higher NfL or a lower ptau/ttau ratio 263 and greater UMN disease burden according to the Penn UMN score (rho=0.4, $p<0.001$, rho=$2640.2, p=0.03 ;$ Figure 2B\&D). 

score.
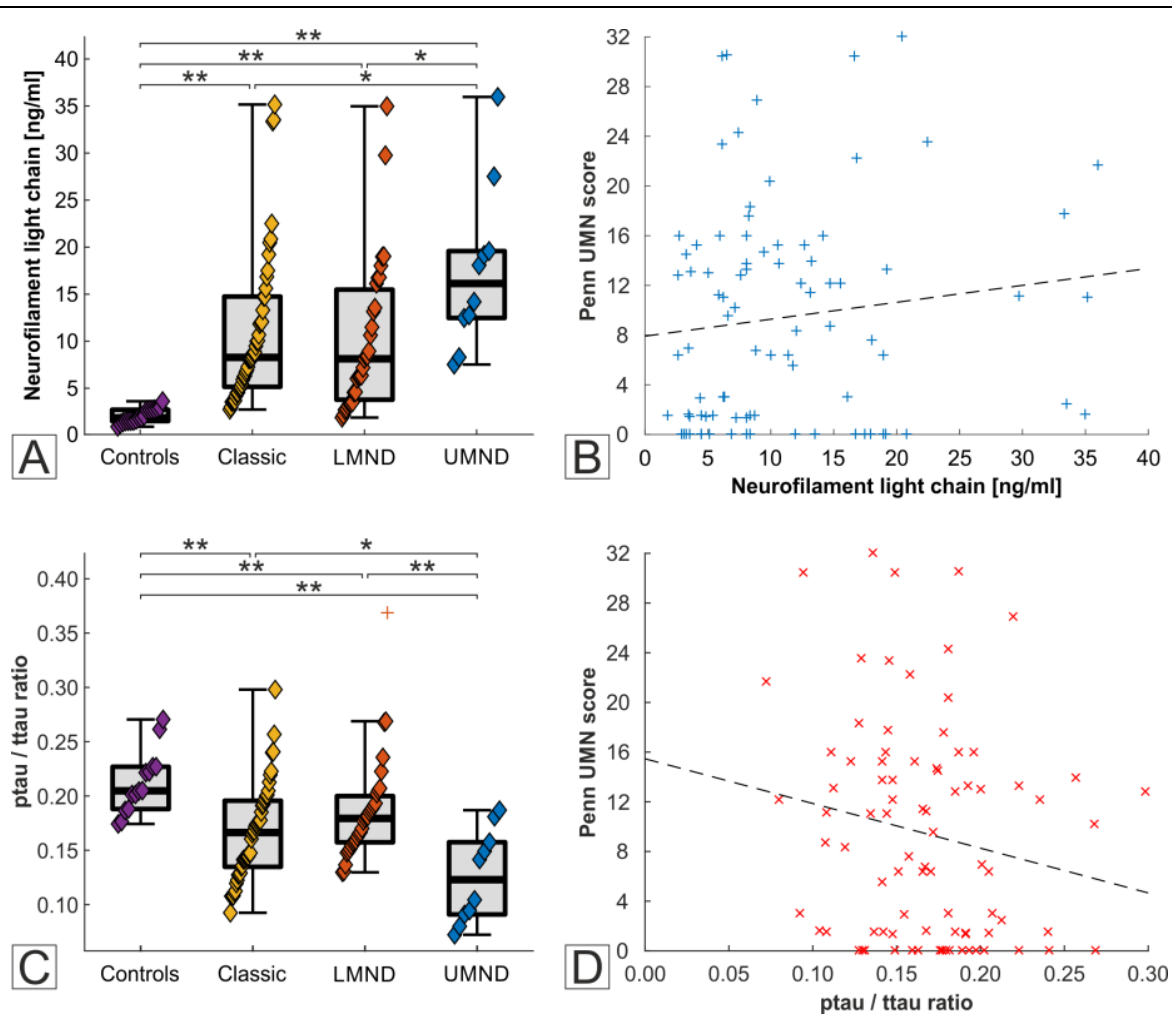

Figure 2. CSF NfL concentrations and the ptau/ttau ratio across the ALS disease spectrum

When compared to classic and lower motor neuron dominant (LMND) ALS, upper motor neuron dominant (UMND) ALS phenotype was related to significantly higher CSF NfL concentrations $(\mathbf{A})$ and a significantly lower ptau/ttau ratio (C). Higher CSF NfL levels and a smaller ptau/ttau ratio were moreover associated with a greater UMN disease burden as assessed by the Penn UMN score (B\&D). ${ }^{*} p \leq 0.05,{ }^{* *} p \leq 0.001$.

CSF NfL, ttau, ptau, the ptau/ttau ratio and biomarkers of neuroaxonal injury in ALS

268 There was a medium-effect size relationship between NfL and median CST FA, MD and RD 269 (ROl-based approach, rho $=-0.5, p=0.001$, rho $=0.3, p=0.02$, rho $=0.5, p<0.001$; Figure 3A\&C). 270 Correlations between NfL and median CST FA and RD remained significant when solely 
271 considering the classic ALS patients (rho $=-0.4, p=0.03$, rho $=0.4, p=0.05$; please see the 272 Supplemental and Supplemental Figure 2). For the whole ALS cohort, strong correlation 273 between $\mathrm{NfL}$ and FA along the cortical spinal pathway additionally emerged from an 274 unbiased whole-brain analysis and it was statistically significant at the stringent threshold of $\mathrm{p}<0.05$ FWE corrected (Figure 3B). At the same statistical threshold, the regression analysis revealed also a positive correlation between NfL and RD which spatially overlapped with the

277 distribution of the significant results in the FA analysis (Figure 3D).

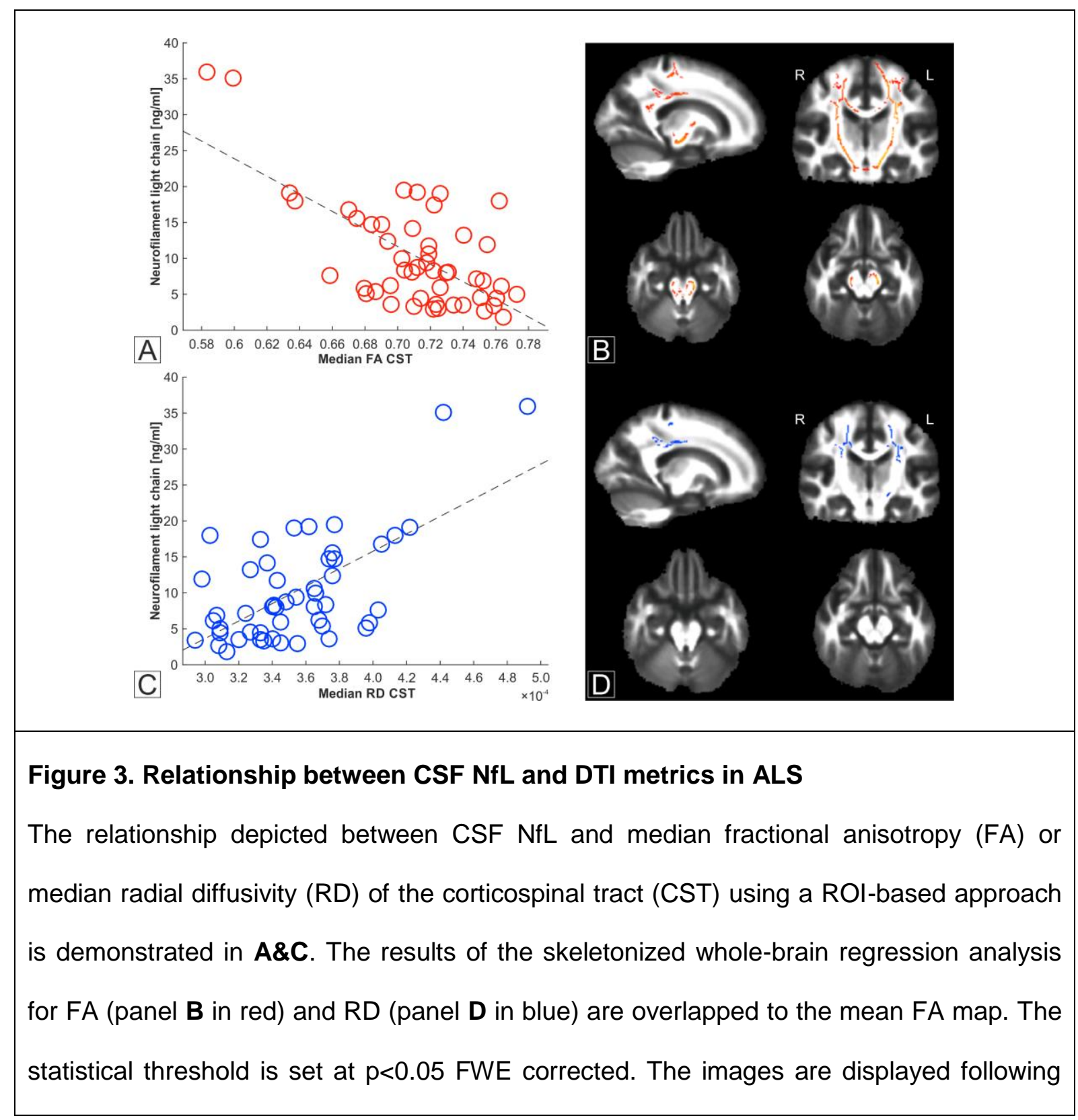


the radiological convention.

278 Likewise, there was a medium-effect size relationship between the ptau/ttau ratio and 279 median CST FA and RD (ROI-based approach, rho=0.4, $p=0.01$, rho=-0.3, $p=0.03$; Figure 280 4A\&C). Considering the whole-brain analysis, the correlation between the ptau/ttau ratio and 281 DTI metrics survived the stringent FWE correction for multiple comparison $(p<0.05)$ and 282 partially overlapped with the results of the correlation analysis between NfL level and DTI 283 metrics (Figure 3B\&D and Figure 4B\&D). In both cases the CST was involved (please see 284 also the results of the $\mathrm{ROI}$ analysis, Figure $3 \mathbf{A} \& \mathbf{C}$, Figure $4 \mathbf{A} \& \mathbf{C}$ ), but the whole-brain 285 analysis revealed that the ptau/ttau ratio was also related to a FA decrease in the genu of the corpus callosum, in the anterior portion of the corona radiata (bilateral), in the anterior portion of the cingulum WM (right), in the external capsule (left) and in anterior limb of the internal capsule (left) (Figure 4B). The ptau/ttau ratio was also correlated with increased RD values 289 in all sections of the corpus callosum (Figure 4D).

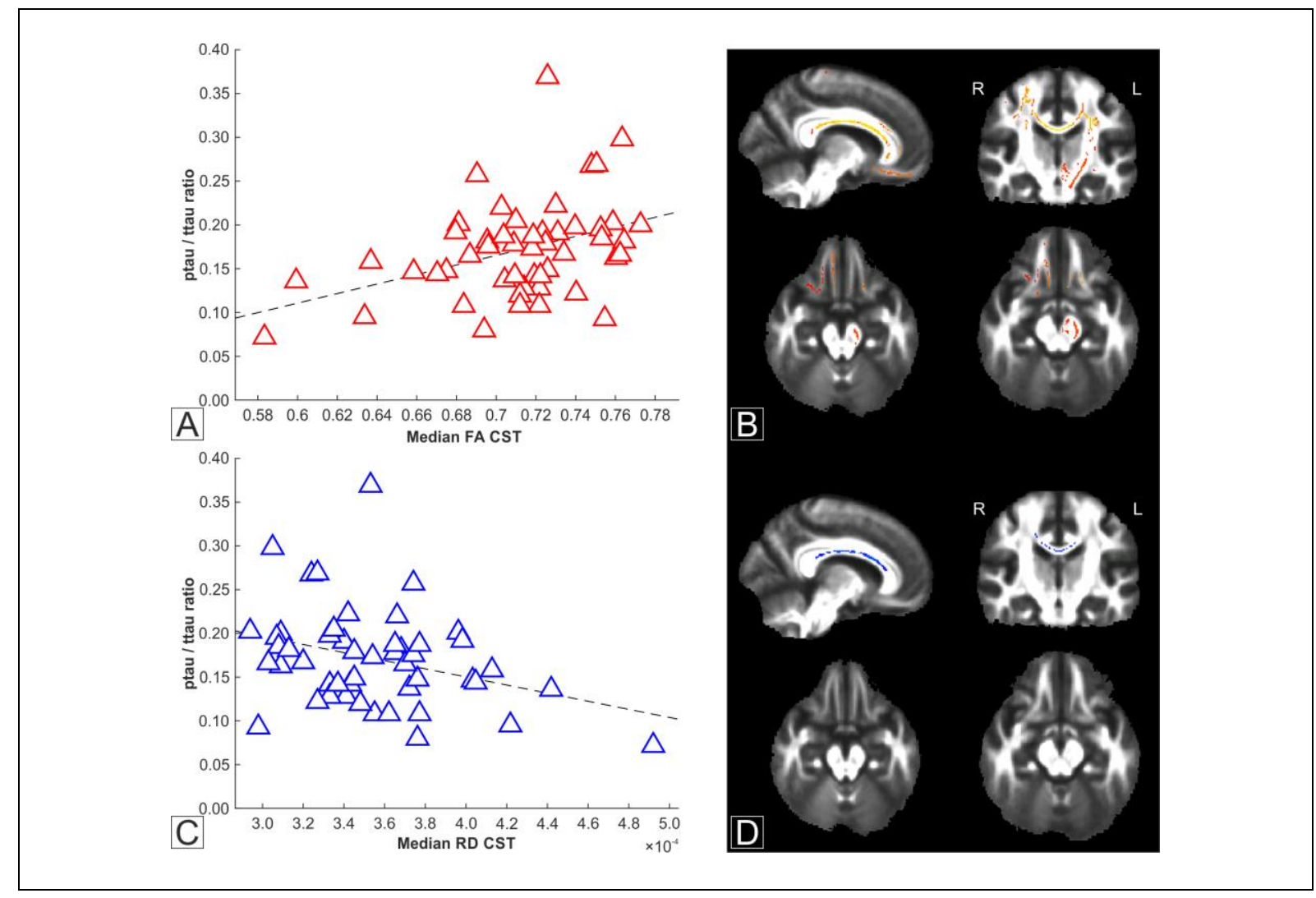




\section{Figure 4. Relationship between the CSF ptau/ttau ratio and DTI metrics in ALS}

The relationship depicted between the CSF ptau/ttau ratio and median FA or RD of the CST using a ROI-based approach is demonstrated in A\&C. The results of the skeletonized whole-brain regression analysis for FA (panel $\mathbf{B}$ in red) and RD (panel $\mathbf{D}$ in blue) are overlapped to the mean FA map. The statistical threshold is set at $p<0.05$ FWE corrected. The images are displayed following the radiological convention.

There was no relationship between NfL, ttau, ptau and the ptau/ttau ratio and $\mathrm{WMH}$.

FAMD revealed a co-variance pattern between CSF NfL (factor load -0.6), the ptau/ttau ratio (0.7), CST FA (0.8) and UMND ALS phenotype (-2.7), which has to be interpreted this way, 293 that high NfL together with a lower ptau/ttau ratio and CST FA decrease is found in patients 294 with dominant UMN involvement.

A lower ptau/ttau ratio was, moreover, related to smaller GMV ( $r=0.3, p=0.02)$. There was no association between CSF NfL, ptau, ttau and the ptau/ttau ratio and any further PNS and CNS axonal or (neuro)degeneration ALS marker (e.g. nerve CSA, CMAP amplitudes, cortical 298 thickness of the precentral gyrus).

\section{CSF NfL, ttau, ptau and the ptau/ttau ratio and long-term prognosis in ALS}

There was a small-effect size inverse relationship between NfL and baseline ALSFRS-R total score (rho=-0.2, $\mathrm{p}=0.03$ ): ALS patients with higher compared to lower $\mathrm{NfL}$ (median-split) revealed lower ALSFRS-R total scores $(H(1)=4.6, p=0.03)$.

Mixed effects linear models displayed a significant NfL main effect on longitudinal ALSFRS-R total score $(e=-4.9, p=0.01)$, while there was no significant $\mathrm{NfL} \times$ time interaction effect. This means that when averaging the ALSFRS-R total score across all available time points, ALS patients with higher compared to lower baseline NfL (median-split) show a -4.9 points lower 307 mean value. 
308 There was a trend-level group effect of slow, intermediate and fast progressors on baseline 309 CSF NfL $(H(2)=5.0, p=0.08)$. Posthoc analysis revealed that fast compared to intermediate 310 progressors displayed significantly higher $\mathrm{NfL}(\mathrm{Z}=2.3, \mathrm{p}=0.02)$ (Figure 5A). In line with this, 311 there was a small-effect size correlation between NfL and DPR (rho=0.2, $\mathrm{p}=0.07$, trend312 level).

313 Cox proportional hazard modelling depicted a 2fold to 3fold greater hazard of death for 314 patients with high CSF NfL compared to patients having medium or low $N f L(\operatorname{Exp}(B)$ $315[95 \% \mathrm{Cl}]=0.5[0.3,0.9], p=0.01, \operatorname{Exp}(B)[95 \% \mathrm{Cl}]=0.3[0.1,0.9], p=0.02)$. Hazard remained 316 after model adjustment for age, sex, onset site, sporadic vs. familial ALS and baseline 317 ALSFRS-R total score $(\operatorname{Exp}(B)[95 \% C l]=0.4[0.2,0.8], p=0.007, \operatorname{Exp}(B)[95 \% \mathrm{Cl}]=0.3[0.1$, $3180.9], p=0.03$; Figure 5B).

319 Kaplan-Meier analysis was in line with those results, displaying significantly shorter median 320 [SE] survival times of patients showing high (upper tercile, 34 [3] months) compared to 321 medium (medium tercile, 48 [3], $\chi^{2}=6.1, p=0.01$ ) or low baseline NfL (lower tercile, 45 [22], 322 $\chi^{2}=5.1, p=0.02 ;$ Figure 5C)

There was no relationship between ttau, ptau or the ptau/ttau ratio and the patients' 324 functional scoring (ALSFRS-R), disease progression and survival.
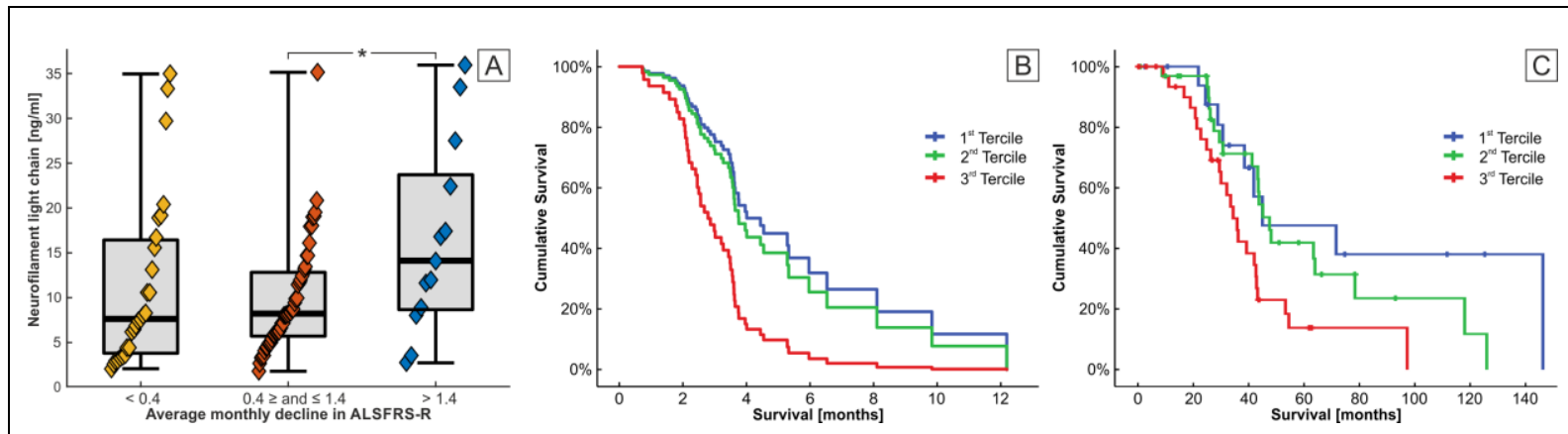

Figure 5. Disease progression and survival as a function of baseline CSF NfL values in ALS 
Fast disease progression (averaged ALSFRS-R points lost per month $>1.4$ ) was related to higher baseline CSF NfL levels (A). B depicts predicted survival curves after covariate adjustment (age, sex, onset site, sporadic vs. familial ALS, baseline ALSFRS-R total score) for CSF NfL terciles (Cox proportional hazard modelling). ALS patients with high (3rd tercile) compared to medium (2nd tercile) and low (1st tercile) baseline NfL levels display a 2fold to 3fold greater hazard of death. C demonstrates Kaplan-Meier analysis; median survival time was significantly shorter in ALS patients exhibiting baseline CSF NfL levels within the upper (3rd) tercile compared to patients revealing baseline CSF NfL concentrations within the medium (2nd) or lower tercile (1st). ${ }^{*} p<0.05$. 


\section{Discussion}

327 Our analysis argues that CSF NfL and the ptau/ttau ratio act as diagnostic biomarkers which 328 at once relate to one another, to UMN involvement and DTI white matter signature of 329 cerebral CST degeneration. A smaller ptau/ttau ratio was further indicative of whole-brain 330 gray matter atrophy and widespread microstructural white matter pathology. Neither NfL nor 331 CSF tau measures were related to peripheral motor axon involvement. Our results, 332 moreover, demonstrate a particular relationship between higher baseline CSF NfL and 333 greater disease severity, more rapid disease progression, greater hazard of death and 334 shorter survival in ALS. These findings suggest that elevated CSF NfL and a lower ptau/ttau 335 ratio are particularly biomarkers of central motor degeneration that together with measures 336 emerging from microstructural white matter neuroimaging could be used to stratify ALS 337 patients and to monitor their disease progression presumably allowing to assess the efficacy 338 of future neuroprotective therapies.

339 Irrespective of ALS pathophysiology, neurofilaments are structural constituents of the neuroaxonal cytoskeleton and integral components of synapses; they are essential for 341 axonal growth, transport and signaling pathways. Neurofilaments are highly abundant in the 342 large Betz cells of the motor cortex and in large-caliber myelinated axons, e.g. of the CST 343 [44-47]. White matter and cortical injury is related to elevated CSF NfL that represents a 344 downstream effect of neuroaxonal loss [48-51]. Tau is a microtubule-associated protein that 345 is highly expressed in neuronal axons, e.g. in thin unmyelinated axons of the neocortical gray 346 matter, providing axonal transport and maintenance of the neurons' structure/morphology $347[52,53]$. Neuroaxonal degeneration results in increased release of tau from the brain into the 348 interstitial fluid/CSF; and - like CSF NfL - elevation of CSF ttau likely reflects unspecific 349 neuronal and axonal damage, as observed in many chronic neurodegenerative diseases 350 [54]. High CSF ptau, however, specifically relates to the occurrence of neurofibrillary tangle 351 formations and is one hallmark diagnostic biomarker of Alzheimer's disease (AD) [55]. 
352 This work replicates the results of several studies showing significantly higher CSF NfL levels 353 in ALS compared to controls (e.g. [4, 6, 14]). Our findings, moreover, support recent data that 354 ALS relates to a lower ptau/ttau ratio, indicating a shifted proportion of ttau and ptau which 355 might be based on three constellations: ALS patients compared to controls reveal (i) higher 356 CSF ttau, or (ii) lower CSF ptau, or both - (iii) higher CSF ttau together with lower CSF ptau. 357 Recent studies reporting a reduced ptau/ttau ratio in ALS or ALS with frontotemporal 358 dementia (ALS-FTD), either disclosed higher CSF ttau [24, 56, 57] or lower ptau [23]. 359 Because phosphorylation of tau occurs mainly in $A D$ and not so much in other 360 neurodegenerative disorders, one may suspect that a lower ptau/ttau ratio probably reflects severe neuroaxonal damage in rapidly progressive diseases favoring the hypothesis of 362 increased CSF ttau rather than a ptau reduction [57]. In our ALS sample, however, both ttau 363 and ptau were unaltered, indicating that the ptau/ttau ratio seems to be a more sensitive 364 candidate biomarker in ALS than CSF ttau or ptau alone.

365 As shown before, in ALS CSF NfL is skewed towards lower levels, leaving the pivotal 366 question what drives the substantial CSF NfL increase found in a certain ALS subgroup. Our 367 data reveal that in ALS the variability of $\mathrm{NfL}$ depends on cerebral CST degeneration and 368 UMN involvement, extending the findings of previous studies $[4,5,16]$ by showing for the 369 first time that those variables co-vary, implying that they are altered together in the same 370 patient. This is supported by the fact that in ALS the strong DTI white matter signature in 371 terms of CST FA decrease also relates to UMN pathology [28, 34, 58-60]. Constellation of 372 high CSF NfL, CST degeneration and dominant UMN involvement additionally goes along 373 with a lower ptau/ttau ratio. Supposing that a smaller ptau/ttau ratio indicates neuroaxonal 374 injury, our findings emphasize that CSF NfL and the ptau/ttau ratio together stand for the 375 extent and the severity of upper motor neuron degeneration in ALS. However, in contrast to $376 \mathrm{NfL}$, a reduced ptau/ttau ratio further indicates gray matter atrophy and white matter integrity 377 loss beyond upper motor neuron pathology. This suggests that the ratio could also serve as 
378 a marker for extramotor involvement in ALS, which needs to be elucidated within future 379 studies.

380 In our sample CSF NfL and the ptau/ttau ratio were not only related to $\mathrm{FA}$, but also to various 381 DTI metrics (MD, RD). FA and RD alterations seem to be sensitive against demyelination, 382 Wallerian-type myelin degeneration and axonal integrity loss, especially in chronic diseases 383 with extended axonal damage; MD changes have, moreover, been speculated to relate to 384 augmented cellularity (e.g. due to the loss of large myelinated axons) [61, 62]. As the 385 biological underpinnings of differences in DTI variables are still unclear, especially in terms of 386 co-existing underlying pathologies (i.e. axonal injury, demyelination, inflammation) [62, 63], 387 we prefer to refrain from further interpretations of those particular findings.

388 Our results are in line with two recent studies relating higher CSF NfL and a lower ptau/ttau 389 ratio to altered DTI metrics in ALS $[16,23]$. They, however, contradict another ALS study that 390 did not find any relationship between CSF NfL and DTI CST integrity [6]. Steinacker et al. 391 therein combine participants scanned on two different MRI systems and with two different 392 field strengths (2/3 underwent a 1.5T MRI, 1/3 underwent a $3 \mathrm{~T} \mathrm{MRI).} \mathrm{The} \mathrm{authors} \mathrm{have}$ 393 shown that the data obtained from the two systems were comparable and they thus 394 combined all the DTI values in a single analysis. A lower field strength (1.5T compared to 3T) 395 inherently entails a lower signal-to-noise ratio that could, potentially, mask the presence of an 396 effect like the relationship between FA values and NfL levels. Our study, as well as the 397 aforementioned study of Menke and colleagues [16], which also reported a correlation 398 between both FA and RD values and levels of NfL in ALS patients, is based on data acquired 399 on a single 3T scanner employing only one protocol. This kind of design is likely to enhance 400 the sensitivity of the study to detect effects that could, otherwise, be masked by noise.

401 One might have expected to find an association between motor cortex thickness denoting 402 UMN pathology and CSF NfL and the ptau/ttau ratio. As Betz cells and their gray matter 
403 axons just represent a small fraction of the motor cortex their degeneration seems to be 404 better mirrored by NfL levels or the ptau/ttau ratio instead of affecting the overall 405 number/density of motor neurons/gray matter neuropil or precentral gyrus thickness, 406 accounting for the absent relationship [42, 64].

407 We failed to find a relationship between CSF NfL or the ptau/ttau ratio, clinical LMN 408 involvement and LMN biomarkers of axonal injury (e.g. reduced CMAP amplitudes or nerve 409 CSA indicating muscle and nerve atrophy, respectively). However, patients with LMND 410 clinical phenotype presenting isolated LMN signs, likewisely displayed elevated NfL 411 concentrations or a reduced ptau/ttau ratio within the range of classic ALS. ALS disease 412 mimics with sole LMN involvement, i.e. e.g. Kennedy's disease or spinal muscular atrophy, 413 do contrary not show abnormal neurofilament levels [5, 14]. Our findings of significant NfL 414 increase across all clinical phenotypes are in line with previous observations in early 415 symptom onset ALS [14], strengthening the role of NfL and ptau/ttau as biomarkers which 416 enhance the diagnostic accuracy of ALS, especially in patients with predominant or isolated 417 LMN signs.

418 Corroborating previous findings, these analyses also indicate that in ALS higher CSF NfL 419 refers to greater disease severity at baseline and longitudinal follow-up. It conversely 420 remains vague whether higher baseline $\mathrm{NfL}$ also relates to a steeper decline or a steady 421 trajectory of overall motor function (group effect of slow, intermediate and fast progressors on 422 NfL vs. non-significant time $\times$ median-split baseline NfL interaction effect on longitudinal 423 ALSFRS-R total score). Considering the latter, it might be possible that a certain ALS subject 424 just comes into the disease with an already determined signature of functional performance 425 and related CSF NfL levels. This would be in line with recent analysis demonstrating no or 426 just minimal change of CSF and highly related serum neurofilament concentrations over the 427 course of disease $[7,11,13]$. Especially at the lower end of CSF NfL levels in ALS, there 428 might be a continuous turnover of neurofilaments as a result of an equilibrium between 24 
429

430

431

432

433

434

435

436

437

438

439

440

441

442

443

444

445

446

447

448

449

450

451

452

453

454

neurodegeneration and -regeneration, with the latter depending on individual variables (e.g. genetics, resilience, exposure to environmental (epigenetic) factors throughout life [43]). Due to such individual circumstances a certain ALS subgroup abandons that equilibrium (supposedly at an already preclinical state or rather suddenly indicating the onset of irreversible neuroaxonal damage $[5,65])$, passing the threshold for disease development and CSF NfL increase which in turn relates to shorter survival. This model especially holds true for the ALS conversion of asymptomatic familial cases revealing normal CSF NfL at presymptomatic but highly elevated levels at symptomatic disease stages [4, 11, 65]. We, however, refrained from comparing NfL between sporadic and familial ALS, because of the small number of genetic variants. Impact of CSF NfL on long-term prognosis remains significant after the adjustment for several disease-modifying variables, replicating previous findings [4, 7] and suggesting $\mathrm{NfL}$ to aid as an independent prognostic biomarker.

The association of the same biomarker with both UMND ALS and worse prognosis is somewhat puzzling, as the UMND phenotype compared to classic ALS usually has a slower functional decline. Our results may thereby point to the existence of distinct groups displaying high CSF NfL: UMND ALS with longer survival despite high CSF NfL and ALS patients with combined UMN and LMN pathology (classic disease phenotype), high CSF NfL and worse prognosis [4] (see follow-up analysis in the Supplemental and Supplemental Figure 3). Further studies are indeed needed to disentangle the existence of such subgroups.

The strength of our study is the availability of a considerable set of locally well-established imaging biomarkers used to understand the co-variance patterns between CSF NfL, CSF tau measures and further measures of PNS and CNS neuroaxonal damage in ALS. Limitations comprise: (i) our cross-sectional and retrospective approach, (ii) the relatively small sample size of distinct clinical ALS subgroups (especially of UMND ALS, making up only $11 \%$ of our patient cohort), which kept us from performing extensive phenotype-wise analysis (of e.g. the 
455 relationship between CSF measures and neuroaxonal injury markers), as well as (iii) the 456 absence of measures of serum NfL and phosphorylated neurofilament heavy chain (pNFH) 457 (e.g. to investigate the superiority of one marker to reflect disease severity and biomarker 458 neuroaxonal injury). An additional limitation of the study is the heterogeneity among the 459 number of patients who underwent each assessment (Supplemental Figure 1).

460 Nevertheless our findings overall strengthen the idea that combining CSF NfL, the ptau/ttau 461 ratio, CST DTI metrics and clinical measures (of e.g. UMN pathology) improve the diagnostic 462 accuracy and prognostic assessment in ALS. 


\section{References}

464

465

466

467

468

469

470

471

472

473

474

475

476

477

478

479

480

481

482

483

484

485

486

487

488

489

490

491

492

493

494

495

496

497

498

499

500

501

502

503

504

505

506

507

508

509

510

511

512

513

1. Meeter LH, Kaat LD, Rohrer JD, van Swieten JC (2017) Imaging and fluid biomarkers in frontotemporal dementia. Nat Rev Neurol 13(7): 406-419. doi: 10.1038/nrneurol.2017.75

2. Mattsson N, Insel PS, Palmqvist S, Portelius E, Zetterberg H, Weiner M, Blennow K, Hansson $O$ (2016) Cerebrospinal fluid tau, neurogranin, and neurofilament light in Alzheimer's disease. EMBO Mol Med 8(10): 1184-1196. doi: 10.15252/emmm.201606540

3. Kaiserova M, Grambalova Z, Otruba P, Stejskal D, Prikrylova Vranova H, Mares J, Mensikova K, Kanovsky P (2017) Cerebrospinal fluid levels of chromogranin A and phosphorylated neurofilament heavy chain are elevated in amyotrophic lateral sclerosis. Acta Neurol Scand (Acta Neurol Scand). doi: 10.1111/ane.12735

4. Gaiani A, Martinelli I, Bello L, Querin G, Puthenparampil M, Ruggero S, Toffanin E, Cagnin A, Briani C, Pegoraro E, Sorarù G (2017) Diagnostic and Prognostic Biomarkers in Amyotrophic Lateral Sclerosis: Neurofilament Light Chain Levels in Definite Subtypes of Disease. JAMA Neurol 74(5): 525-532. doi: 10.1001/jamaneurol.2016.5398

5. Poesen K, Schaepdryver M de, Stubendorff B, Gille B, Muckova P, Wendler S, Prell T, Ringer TM, Rhode H, Stevens O, Claeys KG, Couwelier G, D'Hondt A, Lamaire N, Tilkin P, van Reijen D, Gourmaud S, Fedtke N, Heiling B, Rumpel M, Rödiger A, Gunkel A, Witte OW, Paquet C, Vandenberghe R, Grosskreutz J, van Damme P (2017) Neurofilament markers for ALS correlate with extent of upper and lower motor neuron disease. Neurology 88(24): 2302-2309. doi: 10.1212/WNL.0000000000004029

6. Steinacker P, Feneberg E, Weishaupt J, Brettschneider J, Tumani H, Andersen PM, Arnim CAF von, Böhm S, Kassubek J, Kubisch C, Lulé D, Müller H-P, Muche R, Pinkhardt E, Oeckl P, Rosenbohm A, Anderl-Straub S, Volk AE, Weydt P, Ludolph AC, Otto $M(2016)$ Neurofilaments in the diagnosis of motoneuron diseases: A prospective study on 455 patients. J Neurol Neurosurg Psychiatry 87(1): 12-20. doi: 10.1136/jnnp2015-311387

7. Lu C-H, Macdonald-Wallis C, Gray E, Pearce N, Petzold A, Norgren N, Giovannoni G, Fratta P, Sidle K, Fish M, Orrell R, Howard R, Talbot K, Greensmith L, Kuhle J, Turner MR, Malaspina A (2015) Neurofilament light chain: A prognostic biomarker in amyotrophic lateral sclerosis. Neurology 84(22): 2247-2257. doi: 10.1212/WNL.0000000000001642

8. Steinacker P, Verde F, Fang L, Feneberg E, Oeckl P, Roeber S, Anderl-Straub S, Danek A, Diehl-Schmid J, Fassbender K, Fliessbach K, Foerstl H, Giese A, Jahn H, Kassubek J, Kornhuber J, Landwehrmeyer GB, Lauer M, Pinkhardt EH, Prudlo J, Rosenbohm A, Schneider A, Schroeter ML, Tumani H, Arnim CAF von, Weishaupt J, Weydt P, Ludolph AC, Yilmazer Hanke D, Otto M (2017) Chitotriosidase (CHIT1) is increased in microglia and macrophages in spinal cord of amyotrophic lateral sclerosis and cerebrospinal fluid levels correlate with disease severity and progression. $\mathrm{J}$ Neurol Neurosurg Psychiatry. doi: 10.1136/jnnp-2017-317138

9. Schaepdryver M de, Jeromin A, Gille B, Claeys KG, Herbst V, Brix B, van Damme P, Poesen K (2017) Comparison of elevated phosphorylated neurofilament heavy chains in serum and cerebrospinal fluid of patients with amyotrophic lateral sclerosis. J Neurol Neurosurg Psychiatry. doi: 10.1136/jnnp-2017-316605

10. Chen X, Chen Y, Wei Q, Ou R, Cao B, Zhao B, Shang H-F (2016) Assessment of a multiple biomarker panel for diagnosis of amyotrophic lateral sclerosis. BMC. Neurol. 16: 173. doi: 10.1186/s12883-016-0689-x

11. Gendron TF, Daughrity LM, Heckman MG, Diehl NN, Wuu J, Miller TM, Pastor P, Trojanowski JQ, Grossman M, Berry JD, Hu WT, Ratti A, Benatar M, Silani V, Glass JD, Floeter MK, Jeromin A, Boylan KB, Petrucelli L (2017) Phosphorylated neurofilament 
heavy chain: A biomarker of survival for C9ORF72-associated amyotrophic lateral sclerosis. Ann Neurol. doi: 10.1002/ana.24980

12. Brettschneider J, Petzold A, Süssmuth SD, Ludolph AC, Tumani H (2006) Axonal damage markers in cerebrospinal fluid are increased in ALS. Neurology 66(6): 852-856. doi: 10.1212/01.wnl.0000203120.85850.54

13. Steinacker P, Huss A, Mayer B, Grehl T, Grosskreutz J, Borck G, Kuhle J, Lulé D, Meyer T, Oeckl P, Petri S, Weishaupt J, Ludolph AC, Otto M (2017) Diagnostic and prognostic significance of neurofilament light chain NF-L, but not progranulin and $\mathrm{S} 100 \mathrm{~B}$, in the course of amyotrophic lateral sclerosis: Data from the German MND-net. Amyotrophic Lateral Sclerosis and Frontotemporal Degeneration 18(1-2): 112-119. doi: 10.1080/21678421.2016.1241279

14. Feneberg E, Oeckl P, Steinacker P, Verde F, Barro C, van Damme P, Gray E, Grosskreutz J, Jardel C, Kuhle J, Koerner S, Lamari F, Amador MDM, Mayer B, Morelli C, Muckova P, Petri S, Poesen K, Raaphorst J, Salachas F, Silani V, Stubendorff B, Turner MR, Verbeek MM, Weishaupt JH, Weydt P, Ludolph AC, Otto M (2018) Multicenter evaluation of neurofilaments in early symptom onset amyotrophic lateral sclerosis. Neurology 90(1): e22-e30. doi: 10.1212/WNL.0000000000004761

15. Boylan KB, Glass JD, Crook JE, Yang C, Thomas CS, Desaro P, Johnston A, Overstreet K, Kelly C, Polak M, Shaw G (2013) Phosphorylated neurofilament heavy subunit (pNF-H) in peripheral blood and CSF as a potential prognostic biomarker in amyotrophic lateral sclerosis. J. Neurol. Neurosurg. Psychiatry 84(4): 467-472

16. Menke RA, Gray E, Lu CH, Kuhle J, Talbot K, Malaspina A, Turner MR (2015) CSF neurofilament light chain reflects corticospinal tract degeneration in ALS. Ann. Clin. Transl. Neurol 2(7): 748-755

17. Turner MR, Gray E (2016) Are neurofilaments heading for the ALS clinic? J Neurol Neurosurg Psychiatry 87(1): 3-4. doi: 10.1136/jnnp-2015-311934

18. Jiménez-Jiménez FJ, Hernánz A, Medina-Acebrón $S$, Bustos $F$ de, Zurdo JM, Alonso $H$, Puertas I, Barcenilla B, Sayed Y, Cabrera-Valdivia F (2005) Tau protein concentrations in cerebrospinal fluid of patients with amyotrophic lateral sclerosis. Acta Neurol Scand. 111(2): 114-117. doi: 10.1111/j.1600-0404.2005.00370.x

19. Paladino P, Valentino F, Piccoli T, Piccoli F, La Bella V (2009) Cerebrospinal fluid tau protein is not a biological marker in amyotrophic lateral sclerosis. Eur $\mathrm{J}$ Neurol 16(2): 257-261. doi: 10.1111/j.1468-1331.2008.02405.x

20. Ganesalingam J, An J, Shaw CE, Shaw G, Lacomis D, Bowser R (2011) Combination of neurofilament heavy chain and complement C3 as CSF biomarkers for ALS. J Neurochem 117(3): 528-537. doi: 10.1111/j.1471-4159.2011.07224.x

21. Süssmuth SD, Sperfeld AD, Hinz A, Brettschneider J, Endruhn S, Ludolph AC, Tumani $\mathrm{H}$ (2010) CSF glial markers correlate with survival in amyotrophic lateral sclerosis. Neurology 74(12): 982-987. doi: 10.1212/WNL.0b013e3181d5dc3b

22. Sussmuth SD, Tumani H, Ecker D, Ludolph AC (2003) Amyotrophic lateral sclerosis: disease stage related changes of tau protein and $\mathrm{S} 100$ beta in cerebrospinal fluid and creatine kinase in serum. Neurosci. Lett. 353(1): 57-60

23. Grossman M, Elman L, McCluskey L, McMillan CT, Boller A, Powers J, Rascovsky K, Hu W, Shaw L, Irwin DJ, Lee VM-Y, Trojanowski JQ (2014) Phosphorylated tau as a candidate biomarker for amyotrophic lateral sclerosis. JAMA Neurol 71(4): 442-448. doi: 10.1001/jamaneurol.2013.6064

24. Wilke C, Deuschle C, Rattay TW, Maetzler W, Synofzik M (2015) Total tau is increased, but phosphorylated tau not decreased, in cerebrospinal fluid in amyotrophic lateral sclerosis. Neurobiol Aging 36(2): 1072-1074. doi: 10.1016/j.neurobiolaging.2014.10.019

25. Bourbouli M, Rentzos M, Bougea A, Zouvelou V, Constantinides VC, Zaganas I, Evdokimidis I, Kapaki E, Paraskevas GP (2017) Cerebrospinal Fluid TAR DNA-Binding Protein 43 Combined with Tau Proteins as a Candidate Biomarker for Amyotrophic 
Lateral Sclerosis and Frontotemporal Dementia Spectrum Disorders. Dement Geriatr Cogn Disord 44(3-4): 144-152. doi: 10.1159/000478979

26. Abdelhak A, Junker A, Brettschneider J, Kassubek J, Ludolph AC, Otto M, Tumani H (2015) Brain-Specific Cytoskeletal Damage Markers in Cerebrospinal Fluid: Is There a Common Pattern between Amyotrophic Lateral Sclerosis and Primary Progressive Multiple Sclerosis? Int J Mol Sci 16(8): 17565-17588. doi: 10.3390/ijms160817565

27. Brooks BR, Miller RG, Swash M, Munsat TL (2000) El Escorial revisited: revised criteria for the diagnosis of amyotrophic lateral sclerosis. Amyotroph. Lateral. Scler. Other Motor Neuron Disord. 1(5): 293-299

28. Woo JH, Wang S, Melhem ER, Gee JC, Cucchiara A, McCluskey L, Elman L (2014) Linear associations between clinically assessed upper motor neuron disease and diffusion tensor imaging metrics in amyotrophic lateral sclerosis. PLoS. One. 9(8): e105753. doi: 10.1371/journal.pone.0105753

29. Schreiber S, Abdulla S, Debska-Vielhaber G, Machts J, Dannhardt-Stieger V, Feistner H, Oldag A, Goertler M, Petri S, Kollewe K, Kropf S, Schreiber F, Heinze HJ, Dengler R, Nestor PJ, Vielhaber S (2015) Peripheral nerve ultrasound in amyotrophic lateral sclerosis phenotypes. Muscle Nerve 51(5): 669-675

30. Chio A, Calvo A, Moglia C, Mazzini L, Mora G (2011) Phenotypic heterogeneity of amyotrophic lateral sclerosis: a population based study. J. Neurol. Neurosurg. Psychiatry 82(7): 740-746

31. Shibuya K, Simon NG, Geevasinga N, Menon P, Howells J, Park SB, Huynh W, Noto YI, Vucic S, Kiernan MC (2017) The evolution of motor cortical dysfunction in amyotrophic lateral sclerosis. Clin Neurophysiol 128(6): 1075-1082. doi: 10.1016/j.clinph.2017.03.004

32. Jin X, Jiang J-Y, Lu F-Z, Xia X-L, Wang L-X, Zheng C-J (2014) Electrophysiological differences between Hirayama disease, amyotrophic lateral sclerosis and cervical spondylotic amyotrophy. BMC Musculoskelet Disord 15: 349. doi: 10.1186/1471-247415-349

33. Nodera H, Takamatsu N, Shimatani $Y$, Mori A, Sato K, Oda M, Terasawa $Y$, Izumi $Y$, Kaji R (2014) Thinning of cervical nerve roots and peripheral nerves in ALS as measured by sonography. Clin Neurophysiol 125(9): 1906-1911

34. Menke RA, Korner S, Filippini N, Douaud G, Knight S, Talbot K, Turner MR (2014) Widespread grey matter pathology dominates the longitudinal cerebral MRI and clinical landscape of amyotrophic lateral sclerosis. Brain 137(Pt 9): 2546-2555

35. Machts J, Loewe K, Kaufmann J, Jakubiczka S, Abdulla S, Petri S, Dengler R, Heinze HJ, Vielhaber S, Schoenfeld MA, Bede P (2015) Basal ganglia pathology in ALS is associated with neuropsychological deficits. Neurology 85(15): 1301-1309

36. Albuquerque M de, Branco LMT, Rezende TJR, Andrade HMT de, Nucci A, França MC (2017) Longitudinal evaluation of cerebral and spinal cord damage in Amyotrophic Lateral Sclerosis. Neuroimage. Clin. 14: 269-276. doi: 10.1016/j.nicl.2017.01.024

37. Walhout R, Westeneng H-J, Verstraete E, Hendrikse J, Veldink JH, van den Heuvel MP, van den Berg LH (2015) Cortical thickness in ALS: Towards a marker for upper motor neuron involvement. J Neurol Neurosurg Psychiatry 86(3): 288-294. doi: 10.1136/jnnp2013-306839

38. Smith SM, Jenkinson M, Johansen-Berg $H$, Rueckert D, Nichols TE, Mackay CE, Watkins KE, Ciccarelli O, Cader MZ, Matthews PM, Behrens TEJ (2006) Tract-based spatial statistics: Voxelwise analysis of multi-subject diffusion data. Neuroimage. 31(4): 1487-1505. doi: 10.1016/j.neuroimage.2006.02.024

39. Fazekas F, Chawluk JB, Alavi A, Hurtig HI, Zimmerman RA (1987) MR signal abnormalities at $1.5 \mathrm{~T}$ in Alzheimer's dementia and normal aging. AJR Am. J. Roentgenol. 149(2): 351-356

40. Shen D, Cui L, Fang J, Cui B, Li D, Tai H (2016) Voxel-Wise Meta-Analysis of Gray Matter Changes in Amyotrophic Lateral Sclerosis. Front Aging Neurosci. 8: 64 
41. Husson F, Le S, Pages J (2011) Exploratory Multivariate Analysis by Example Using R. CRC Press Taylor \& Francis Group, Boca Raton, FI

42. Cardenas-Blanco A, Machts J, Acosta-Cabronero J, Kaufmann J, Abdulla S, Kollewe K, Petri S, Schreiber S, Heinze HJ, Dengler R, Vielhaber S, Nestor PJ (2016) Structural and diffusion imaging versus clinical assessment to monitor amyotrophic lateral sclerosis. Neuroimage. Clin. 11: 408-414

43. Al Chalabi A, Hardiman O (2013) The epidemiology of ALS: a conspiracy of genes, environment and time. Nat. Rev. Neurol. 9(11): 617-628

44. Oberstadt M, Claßen J, Arendt T, Holzer M (2017) TDP-43 and Cytoskeletal Proteins in ALS. Mol Neurobiol. doi: 10.1007/s12035-017-0543-1

45. Petzold A (2005) Neurofilament phosphoforms: Surrogate markers for axonal injury, degeneration and loss. J Neurol Sci 233(1-2): 183-198. doi: 10.1016/j.jns.2005.03.015

46. Eisen A, Weber $M(2001)$ The motor cortex and amyotrophic lateral sclerosis. Muscle Nerve 24(4): 564-573

47. Yuan A, Sershen H, Veeranna, Basavarajappa BS, Kumar A, Hashim A, Berg M, Lee J$H$, Sato Y, Rao MV, Mohan PS, Dyakin V, Julien J-P, Lee VM-Y, Nixon RA (2015) Neurofilament subunits are integral components of synapses and modulate neurotransmission and behavior in vivo. Mol Psychiatry 20(8): 986-994. doi: $10.1038 / \mathrm{mp} .2015 .45$

48. Zetterberg H, Skillbäck T, Mattsson N, Trojanowski JQ, Portelius E, Shaw LM, Weiner MW, Blennow K (2016) Association of Cerebrospinal Fluid Neurofilament Light Concentration With Alzheimer Disease Progression. JAMA Neurol 73(1): 60-67. doi: 10.1001/jamaneurol.2015.3037

49. Jonsson M, Zetterberg $H$, van Straaten E, Lind K, Syversen S, Edman A, Blennow K, Rosengren L, Pantoni L, Inzitari D, Wallin A (2010) Cerebrospinal fluid biomarkers of white matter lesions - cross-sectional results from the LADIS study. Eur J Neurol 17(3): 377-382. doi: 10.1111/j.1468-1331.2009.02808.x

50. Sjögren $M$, Blomberg $M$, Jonsson $M$, Wahlund LO, Edman A, Lind K, Rosengren L, Blennow K, Wallin A (2001) Neurofilament protein in cerebrospinal fluid: A marker of white matter changes. J. Neurosci. Res. 66(3): 510-516. doi: 10.1002/jnr.1242

51. Brureau A, Blanchard-Bregeon V, Pech C, Hamon S, Chaillou P, Guillemot J-C, Barneoud P, Bertrand P, Pradier L, Rooney T, Schussler N (2017) NF-L in cerebrospinal fluid and serum is a biomarker of neuronal damage in an inducible mouse model of neurodegeneration. Neurobiol Dis 104: 73-84. doi: 10.1016/j.nbd.2017.04.007

52. Ballatore C, Lee VM-Y, Trojanowski JQ (2007) Tau-mediated neurodegeneration in Alzheimer's disease and related disorders. Nat Rev Neurosci 8(9):663-672. doi: $10.1038 / n r n 2194$

53. Trojanowski JQ, Schuck T, Schmidt ML, Lee VM (1989) Distribution of tau proteins in the normal human central and peripheral nervous system. J. Histochem. Cytochem. 37(2): 209-215. doi: 10.1177/37.2.2492045

54. Blennow K, Zetterberg H, Fagan AM (2012) Fluid biomarkers in Alzheimer disease. Cold Spring Harb Perspect Med 2(9): a006221. doi: 10.1101/cshperspect.a006221

55. Zetterberg H (2017) Review: Tau in biofluids - relation to pathology, imaging and clinical features. Neuropathol Appl Neurobiol 43(3): 194-199. doi: 10.1111/nan.12378

56. Meeter LHH, Vijverberg EG, Del Campo M, Rozemuller AJM, Donker Kaat L, Jong FJ de, van der Flier WM, Teunissen CE, van Swieten JC, Pijnenburg YAL (2018) Clinical value of neurofilament and phospho-tau/tau ratio in the frontotemporal dementia spectrum. Neurology 90(14): e1231-e1239. doi: 10.1212/WNL.0000000000005261

57. Pijnenburg YAL, Verwey NA, van der Flier WM, Scheltens P, Teunissen CE (2015) Discriminative and prognostic potential of cerebrospinal fluid phosphoTau/tau ratio and neurofilaments for frontotemporal dementia subtypes. Alzheimers Dement (Amst) 1(4): 505-512. doi: 10.1016/j.dadm.2015.11.001 
58. Menke RAL, Abraham I, Thiel CS, Filippini N, Knight S, Talbot K, Turner MR (2012) Fractional anisotropy in the posterior limb of the internal capsule and prognosis in amyotrophic lateral sclerosis. Arch Neurol 69(11): 1493-1499. doi: 10.1001/archneurol.2012.1122

59. Borsodi F, Culea V, Langkammer C, Khalil M, Pirpamer L, Quasthoff S, Enzinger C, Schmidt R, Fazekas F, Ropele S (2017) Multimodal assessment of white matter tracts in amyotrophic lateral sclerosis. PLoS. One. 12(6): e0178371. doi: 10.1371/journal.pone.0178371

60. Rosenbohm A, Müller H-P, Hübers A, Ludolph AC, Kassubek J (2016) Corticoefferent pathways in pure lower motor neuron disease: A diffusion tensor imaging study. $\mathrm{J}$ Neurol 263(12): 2430-2437. doi: 10.1007/s00415-016-8281-2

61. Acosta-Cabronero J, Nestor PJ (2014) Diffusion tensor imaging in Alzheimer's disease: insights into the limbic-diencephalic network and methodological considerations. Front Aging Neurosci 6: 266. doi: 10.3389/fnagi.2014.00266

62. Winklewski PJ, Sabisz A, Naumczyk P, Jodzio K, Szurowska E, Szarmach A (2018) Understanding the Physiopathology Behind Axial and Radial Diffusivity Changes-What Do We Know? Front Neurol 9: 92. doi: 10.3389/fneur.2018.00092

63. Wheeler-Kingshott CAM, Cercignani M (2009) About "axial" and "radial" diffusivities. Magn Reson Med 61(5): 1255-1260. doi: 10.1002/mrm.21965

64. Toft MH, Gredal O, Pakkenberg B (2005) The size distribution of neurons in the motor cortex in amyotrophic lateral sclerosis. J Anat 207(4): 399-407. doi: 10.1111/j.14697580.2005.00465.x

65. Weydt P, Oeckl P, Huss A, Müller K, Volk AE, Kuhle J, Knehr A, Andersen PM, Prudlo J, Steinacker P, Weishaupt JH, Ludolph AC, Otto M (2016) Neurofilament levels as biomarkers in asymptomatic and symptomatic familial amyotrophic lateral sclerosis. Ann Neurol 79(1): 152-158. doi: 10.1002/ana.24552 
699 Table 1. Demographics and clinical data of the sample under investigation

\begin{tabular}{|c|c|c|c|}
\hline & $\begin{array}{l}\text { ALS } \\
(n=89)\end{array}$ & $\begin{array}{l}\text { Controls } \\
(\mathrm{n}=33)\end{array}$ & P-value \\
\hline Age, in years & $63(33-83)$ & $60(32-76)^{*}$ & 0.06 \\
\hline Male sex, $\mathrm{n}(\%)$ & $54(61)$ & $16(49)^{\#}$ & 0.3 \\
\hline Sporadic ALS / Familial ALS, n (\%) & $63(91) / 6(9)^{1}$ & & \\
\hline $\begin{array}{l}\text { El Escorial na / suspected / possible / } \\
\text { probable / definite, } \mathrm{n}(\%)\end{array}$ & $\begin{array}{l}1(1) / 24(27) / 34 \\
(38) / 15(17) / 15(17)\end{array}$ & & \\
\hline $\begin{array}{l}\text { Clinical phenotypes classic / LMND / } \\
\text { UMND / PLS, } \mathrm{n}(\%)\end{array}$ & $\begin{array}{l}46(52) / 31(35) / 10 \\
(11) / 2(2)\end{array}$ & & \\
\hline Disease onset bulbar / limb, n (\%) & $29(33) / 60(67)$ & & \\
\hline Disease duration, in months & $10(0.2-190)$ & & \\
\hline Disease progression rate, in $1 /$ months & $0.7(0.04-3.3)$ & & \\
\hline ALSFRS-R total score / 48, baseline & $41(4-48)$ & & \\
\hline
\end{tabular}

700 Unless otherwise reported, medians and (ranges) are given. ALS, amyotrophic lateral 701 sclerosis; ALSFRS-R, revised ALS functional rating scale; LMND, lower motor neuron 702 dominant; na, not applicable; PLS, primary lateral sclerosis; UMND, upper motor neuron 703 dominant; ${ }^{1}$ familial ALS comprised 2 cases with C9orf72 positivity and 4 patients with SOD1 704 positivity, *Mann-Whitney $U$ test, ${ }^{\#} \chi^{2}$ test. P-values $<0.05$ were deemed to be statistically 705 significant. 